\title{
A High-Speed Computer for Predicting Radioactive Fallout
}

\author{
J. H. Wright, L. Taback, and H. K. Skramstad
}

\begin{abstract}
A very high-speed analog computer has been developed for predicting the geographic pattern of radioactive fallout that will occur following a nuclear-weapon explosion. Input data consist of wind magnitudes and directions as functions of initial height in the cloud, and the distribution of radioactivity in the cloud as a function of the particle size (in terms of settling rate) and initial height in the cloud. Output data are presented on a cathoderay tube, so that the luminance at any point on the screen represents the intensity of fallout accumulated at that point.
\end{abstract}

\section{Introduction}

The computer described in this paper was developed for the U. S. Weather Bureau and the Albuquerque Operations Office of the Atomic Energy Commission, with the immediate objective of operation in the Pacific Proving Grounds during the 1956 tests of nuclear weapons. Two essentially identical units were built and used by the falloutprediction group as aids for safety purposes. These units, as reported here, were experimental models lacking engineering refinements. A quite different type of special-purpose analog computer, developed by the Sandia Corp., was also emploved. Another machine, using mechanical-optical techniques, is under development at the Los Alamos Scientific Laboratory. The most distinctive features of the National Bureau of Standards machine are its virtually instantaneous speed of solution, straightforward procedure for setting in data, and adaptability to variations of the "model" of the radioactive cloud. Various limitations, as well as some possible refinements, are discussed.

In the past, hand methods of solution have been used extensively. These require considerable simplification of the problem and exploitation of the operator's judgment to achieve the solution in a reasonable time, e. g., an hour or less. Very large digital computing facilities have also been used with considerable reduction in the computation time. Both of these techniques will be of continuing importance; the hand methods, because they require negligible outlay for equipment and installation; the large digital computers, because they can do increasingly complicated problems simply by taking more time. In contrast, the desired objectives for the special-purpose computer were:

1. Small size and reasonable portability.

2. Low cost-under $\$ 10,000$.

3. Problems set up without the need for mathematical training in numerical analysis or digital programing. Actually, the operator need not be familiar even with mathematical functions such as cosine or exponential.

4. Very rapid solution of the problem.

5. Direct visual presentation of the final solution.

6. Rapid problem modification.
These requirements have generally been met in the first model, with some reservations. The solution time is considered to be "instantaneous" because it is negligible compared to the time required for human observation of the output display of data. The estimate of cost is crude and is based on construction of 3 or 4 duplicate machines, exclusive of engineering time or test or of commercial profit. The over-all accuracy of the machine has not been studied systematically, because of the immediate need for field service, and cannot be discussed. The most serious source of error was the conventional cathode-ray tube employed, which, however, provided the most appealing and useful feature of its operation in the first application.

It should be noted at the outset that this computer is not a direct simulation type of analog machine, but rather a scheme for using analog techniques to mechanize the problem in a manner somewhat similar to the organization of the problem in a large digital computer. In terms of apparatus, this machine consists primarily of function generators and scanning voltages. Time, as represented in the flow of machine operations, has no relation to the time variable in the original physical model, but is used for sequencing purposes only.

\section{The Fallout Problem}

The assumptions made in the solution of the problem are not unusual in the field of fallout calculations, and will be listed here in some detail. First, it is assumed that a cloud of radioactive particles has formed, with a definite shape and size, and, at some instant considered as zero time for the calculations, the various kinds of particles at various locations in the cloud begin to settle, being displaced laterally by the winds as they fall to the ground. This ignores the possibility that some particles, notably very large ones, may have reached their maximum height and started falling while other types of dust particles are still rising toward their "initial" positions. The cloud is assumed to have a vertical axis of symmetry, whereas actually the horizontal winds can tilt and otherwise distort the cloud while it is forming. Vertical winds are neglected. The large-scale vortex motions caused by the forces developing the cloud, and any effects on 
the wind pattern resulting from the energy of the burst, are also neglected. It is assumed that the characteristics of the particles are sufficiently described by specifying a rate of fall as a function of altitude, and a particular weighted value of radioactivity for each of the various ranges of fall rate and initial height. We will hereafter refer to a "slowness" variable, $s$, because the corresponding variable in the machine is proportional to fall time rather than rate. The slowness varies greatly with particle size. However, it is assumed that the relative times spent in the various altitude layers are the same for all particles starting from the same height. Thus, the law of fall can be made correct for some intermediate size of particle, but becomes increasingly incorrect toward the extremes of particle size.

Some of the above assumptions become increasingly important for calculations at positions nearing ground zero, the site of the explosion. Eventually, perhaps within an area of two mushroom diameters centered on ground zero, these factors pretty well invalidate the detailed computations. 'The mushroom diameter refers to the mean diameter of the widest portion of the particular cloud considered.

A basic assumption is that the wind at each level is invariant over the entire area of fallout, and does not change during the entire time of fallout. Time and space variability of winds therefore introduce increasingly large errors for particles taking a long time to fall, and for fallout far from ground zero. The relative importance of such variations during actual operations is the subject of present research by Sherman, ${ }^{1}$ Nagler, ${ }^{2}$ and others. Detailed calculations of the effects of variability at all altitudes and areas, and during the time of fallout, could complicate the problem enormously-by several orders of magnitude. Thus, practical considerations of operating conditions become all important, as to whether the requisite detailed weather data can actually be obtained, and soon enough to be valid. For example, if a fallout prediction is to be made 12 $\mathrm{hr}$ before detonation of a bomb, and must allow for particles requiring $24 \mathrm{hr}$ to reach the ground, a very detailed $36-\mathrm{hr}$ weather forecast is necessary. Such forecasting is difficult, if not impossible. Roughly speaking, we may say that beyond about a 200 -mile radius, or 12 -hr falltime, fallout predictions neglecting variability are of doubtful validity, at least without extensive reappraisal by weather experts.

The radial distribution of radioactivity at any one initial height in the cloud is assumed to be the same for all types of particles. As will be seen, the present computer does not control this radial distribution closely. Thus, it may be roughly uniform, or fall off with increasing radius, depending on whether or not a compensating waveform is applied to the intensity grid of the cathode-ray tube. (An arbitrary profile could be generated, but such a refinement was considered unimportant in the first model.) Finally, it is assumed that no diffusion occurs during

\footnotetext{
${ }^{1}$ Los Alamos Scientific Laboratories.
}

2 U. S. Weather Bureau. fallout, so that particles of a given size originating in any specified layer of the cloud maintain their relative positions as they fall. Thus, the calculation of wind effects on particles on the axis is sufficient; i. e., the entire "slice" of cloud (considering particles of one size range) moves as a piece during the fallout.

The machine does not need to distinguish between the number of particles per unit volume, $\rho_{i}$, and the activity per particle, $q_{i}$, for particles of a given size (or "slowness"), $i$. The machine input known as activity, $\alpha$, is the weighted activity for that size of particle for an entire layer of cloud. Thus, $\alpha_{i j}=$ $q_{i j} \rho_{i j} V_{j}$, where the subscript $j$ refers to the $j$ th layer of cloud. $V_{j}$ is the volume of this layer, so that $\rho_{i j} V_{j}$ is the total number of particles in the $j$ th layer of cloud. For a given type of nuclear weapon, values must be assigned to the various $q_{i j}, \rho_{i j}$ and $V_{j}$, so as to define a function of two variables, $\alpha=f(h, s)$, where $h$ represents height in the initial cloud, and $s$ the slowness (time to fall a unit height) typical of a given class of particle. This function $\alpha$ is commonly called the "cloud activity model." In practice, the clend activity model can be supplied to the machine operator as a family of curves, and he need not even be familiar with mathematical functions. The activity function is set up so that the output is in terms of percentage of total activity in the cloud. The family of curves are adjusted with $h$ as a parameter for $0,20,40,60,80$, and 100 percent $^{3}$ of the maximum altitude of the cloud. This normalizing procedure provides for solutions over a considerable range of weapon sizes without readjustment of the cloud activity model as long as the shapes of the clouds remain the same.

It must be noted that the machine does not make any detailed allowance for decay in radioactivity, either during the time of fall or afterward. The assignment of $\alpha_{i j}$ to a given range of particles at a given initial height must include the allowance for decay. Furthermore, for a particular activity model set up in the machine, the operator cannot make a simple single adjustment to show the fallout at some arbitrary earlier stage, i. e., before all the particles included in the model have reached the ground. However, the activity model can be modified to include only those particles that will reach the ground before the specified time. In any case, the model can be set up on either of the following bases:

Integrated dose. The total dosage from time of deposition to some specified time, such as $48 \mathrm{hr}$., or 10 years, after the burst. Often the "infinite dose," for complete decay, is used in estimating possibility of hazard to permanent residents in an area. The unit of measure is the roentgen, $\mathrm{R}$, or milliroentgen, $\mathrm{mr}$.

Dose rate. The intensity of radiation at some selected time after fallout, such as $48 \mathrm{hr}$., This quantity may be expressed in roentgens or milliroentgens per hour, and is appropriate to consideration of evacuating personnel or exploring a con-

${ }^{3}$ Actually, the altitudes must be chosen to correspond to these percentages of total time of fall, and will not be exactly 20,40,60, and 80 percent of the cloud height. 
taminated area. It is a little more convenient than the integrated dose in deriving a model, because the time of arrival need not be known. In practice, it is common to use one law of decay applying to all the batches of particles, the gross fission-product decay expression, $A=A_{0} t^{-1.2}$, in which $A$ is the dose rate measured at time $t$ after the explosion. $A_{0}$ is the dose rate at unit time, such as roentgens per hour at $1 \mathrm{hr}$. On this basis, the completed fallout colculations for dose rates, at some time such as $48 \mathrm{hr}$. after the explosion, can be converted by a single factor to provide the dose rates at some other time, such as 10 days later.

Despite this simplification of the problem, the required amount of computation for a nearly instantaneous presentation is quite formidable. To illustrate this, let us consider the following breakdown of the problem into discrete elements appropriate to digital computation. The cloud is divided into 20 layers vertically, and 25 elementary crosssectional areas in each laver. Thus, there are 500 elemental volumes in the cloud, each containing a distribution of particle sizes, with attendant variations in "slowness" of fall. If we divide this range of particles into 10 classes, there are effectively 5,000 lumps of material in the cloud, each requiring calculation of displacement by the wind in each wind layer through which it falls. On the average, 10 multiplications must be performed to obtain the final horizontal displacement of each elementary lump of material as it falls to the ground, each the product of a time of fall and a wind vector. If the solution is to be completed within $1 / 10$ sec, the required computing rates are:

(a) 50,000 function table values per second, for radioactivity.

(b) 500,000 simple multiplications per second, for partial displacements.

(c) Appropriate combination of these partial results, and presentation on a map.

The display device would be required to generate "picture elements" of varying intensity at the rate of $50,000 / \mathrm{sec}$, one for each representative lump of radioactive dust at its final fallout location. These picture elements tend to be superimposed, because light particles originating in a given layer will fall on top of heavier particles originating from lower altitudes in positions relatively "downwind" with respect to the light particles. This situation will be referred to as overlap. Thus, the display device must be able to accumlate these overlapping contributions, if the computer proper is to be relieved of this burden.

The accuracy requirements on individual operations are rather low, generally ranging from errors of 2 to 10 percent. An analog technique that endeavors to meet approximately the requirements of the example will now be described.

\section{Wind Computations}

Let us assume that the mushroom cloud (see fig. 1) is divided into horizontal layers at graded intervals of

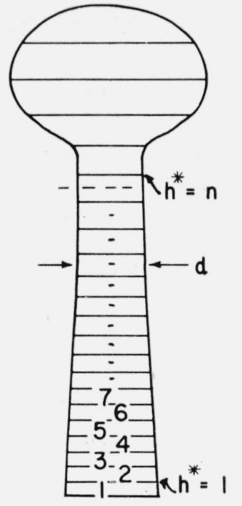

Figure 1. Mushroom cloud.

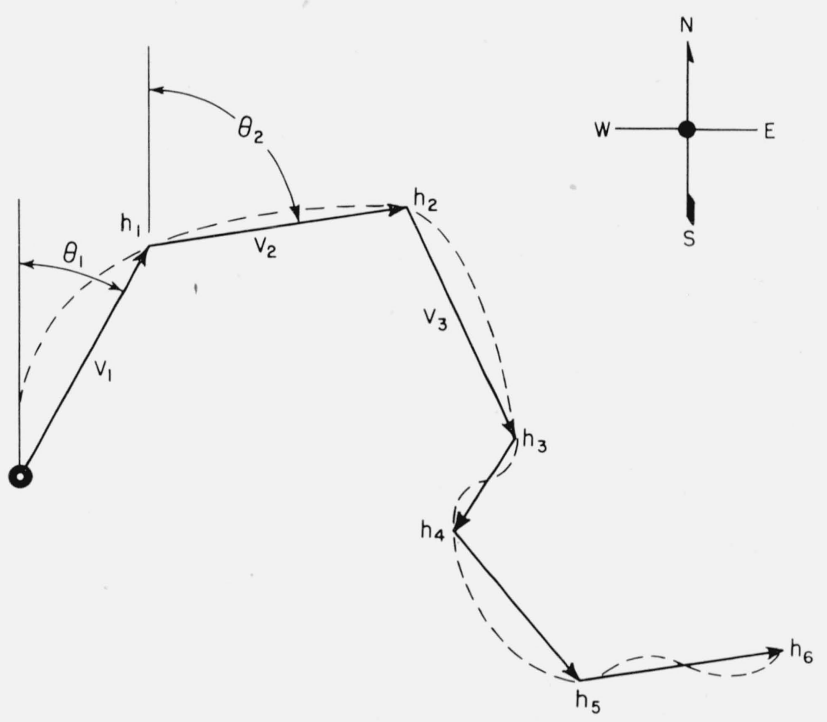

Figure 2. Wind hodograph.

altitude such that a particle of typical size spends equal times falling through successive layers. Let $h$ represent the initial height of any particle. Now let us define a variable $h^{*}$, a function of $h$, which is proportional to the time for a particle to fall from any specified height to the ground. The variable $h^{*}$ has the value 1 at the top of the bottom layer, and $n$ at the top of the $n$th layer. Let $V_{i}$ represent the average speed and $\theta_{i}$ the average wind direction in the $i$ th layer, $\theta$ being measured clockwise from north. The cloud diameter as a function of height is represented by $d(h)$.

Figure 2 shows a wind hodograph. The dotted line may be considered to represent the horizontal projection of the path of a hypothetical weather balloon having a constant rate of ascent. If an appropriate altitude scale is marked along such a curve, a vector such as $V_{2}$ joining altitude points $h_{1}$ and $h_{2}$ would represent the direction and magnitude of the average horizontal wind exerted on this hypothetical balloon as it rises completely through 
the layer between heights $h_{1}$ and $h_{2}$. Such hodographs are commonly used to obtain measurements of winds aloft. In the case of the descending motion of particles, tbe hodograph would be drawn in the reverse sequence, starting with $V_{6}$ instead of $V_{1}$. However, the total relative displacement would be the same, if the time spent in each layer were the same as for the balloon. The horizontal displacement of a particle while it falls from $h_{2}$ to $h_{1}$ is obtained by multiplying this wind vector by the time required for this particular particle to fall through this layer. A particle of size $a$ falling from the top of the third layer to the ground would be displaced in an east-west direction by the amount

$$
x=s(a)\left[V_{1} \sin \theta_{1}+V_{2} \sin \theta_{2}+V_{3} \sin \theta_{3}\right],
$$

where $s(a)$ is the slowness for this size of particle, the time to fall any one height interval. A similar cosine expression holds for the north-south component of displacement.

It is apparent that there is an approximation introduced by assuming a finite number of wind layers, because the dotted line of the wind hodograph deviates from the straight-line vectors joining the layer boundary points $h_{1}, h_{2}$, etc. However, this introduces no error for the particles falling completely through a given layer, because the total effect of the wind is specified correctly. There is a small error, within the layer only, for those particles that start to fall from some height within the particular layer under consideration, because the analog computer interpolates along the straight line in space. If a digital computer were used, it would be necessary to divide the cloud into a great number of layers, or to provide an interpolation routine.

The basic equations for the final ground position of a particle of slowness $s_{j}$, originating on the axis of the cloud within a layer for which $n<h^{*}<n+1$, are therefore

$$
\begin{aligned}
& x=s_{j}\left[\sum_{i=1}^{n} V_{i} \sin \theta_{i}+\left(h^{*}-n\right) V_{n+1} \sin \theta_{n+1}\right] \\
& y=s_{j}\left[\sum_{i=1}^{n} V_{i} \cos \theta_{i}+\left(h^{*}-n\right) V_{n+1} \cos \theta_{n+1}\right],
\end{aligned}
$$

where $x$ represents the fall position east of the explosion site and $y$ the position north of the explosion site. In each equation, the second term, such as $\left(h^{*}-n\right) V_{n+1} \sin \theta_{n+1}$, represents the initial displacement of the particle while falling through a fraction of layer $(n+1)$ enroute to the top of layer $n$.

\section{Description of the Computer}

A simple block diagram of the apparatus is shown in figure 3. From the viewpoint of the operator, there are four basic units:

(a) The winds unit, with knobs for setting in the magnitudes and directions of the mean winds in each of the 20 layers.

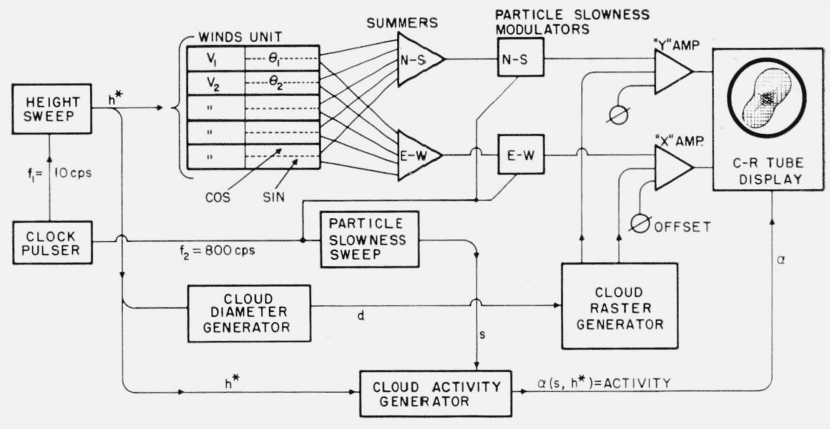

Figure 3. Block diagram of computer.

(b) The cloud geometry unit, including the cloud diameter and cloud raster functions. There are several knobs for adjusting the cloud shape, and one knob for setting in an over-all scale factor for cloud size.

(c) The cloud activity unit, which has 36 knobs for setting in the "model" of distribution of radioactivity in the cloud.

(d) The display unit, which has $x$ and $y$ scale-factor controls (marked $y$-amp and $x$-amp), a brightnesslevel control, and a brightness-slope control.

There is also a central control unit containing all timing and sweep circuits, summing amplifiers, slowness modulators, and final $x$ and $y$ driver amplifiers. It needs no attention in normal operation. In addition, there is a photometer used to measure the brightness on the screen as a direct measure of fallout. It is an electrically and mechanically separate entity.

For explanation, let us consider first that the cloud is collapsed laterally so that all the particles are on the vertical axis of the cloud. Furthermore, consider that we are dealing only with the lightest (i. e., slowest) particles given any consideration in the problem. We will later modify the solution to include heavier particles, and those off the axis of the cloud. A periodic sawtooth voltage is generated to represent the variable $h^{*}$. This is called the height sweep. The voltage rises linearly with time to a nominal $+100 \mathrm{v}$, is reset to zero in about $40 \mu \mathrm{sec}$, and repeats each 1/10 sec, scanning through the full range of $h^{*}$ each time. This is the basic cycle of computer operation, and the entire solution is generated anew with each sweep through the full cloud height. This voltage is applied to the winds unit, a function generator with 20 elements sequentially activated as the input voltage rises, but with their outputs added together at all times. At the start, with the $h^{*}$ voltage at 0 , none of the elements produces an output. As the $h^{*}$ voltage rises from 0 to a nominal value of $+5 \mathrm{v}$, the output of the No. 1 element, representing the lowest wind layer, also rises from 0 to +5 v. As $h^{*}$ rises from +5 to $+10 \mathrm{v}$, the output of element 2 becomes activated, and rises from 0 to $+5 \mathrm{v}$, thereafter remaining at +5 . This goes on until $h^{*}$ reaches a nominal value of $+100 \mathrm{v}$, and all 20 function-generator elements are producing outputs. 


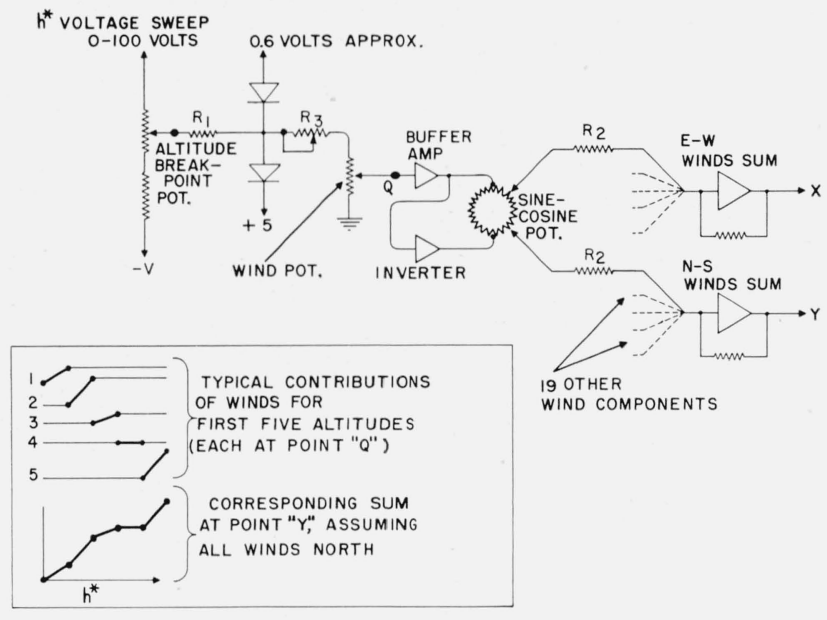

Figure 4. Schematic of winds unit.

This sequential and cumulative action provides the necessary effect of only 1 wind contributing to the total displacement of particles falling from the top of the first layer, but 20 winds contributing to the total effect on particles originating at the very top of the cloud. For a particle originating, say, halfway in layer $n$, the cumulative wind effect is represented by the sum of the earlier sustained voltages, representing lower altitude winds, plus half the full scale voltage from the partly activated $n$th element. The situation is shown in figure 4 for particles in the first five layers of altitude. Actually, before these voltages are summed, they pass through attenuating potentiometers, adjusted according to the mean magnitude of the wind in each layer. The nominal $+5 \mathrm{v}$ represents a maximum-scale wind, e. g., 100 knots. If there is no wind in a given layer, that potentiometer is simply set to zero. The voltages are also resolved into $x$ and $y$ components by sine-cosine potentiometers set according to the wind direction, one for each layer.

Figure 4 also provides a simplified electrical diagram of one element, for one wind layer. When $h^{*}$ is 0 at the beginning of the sweep, the lower diode, connected to a nominal $+5 \mathrm{v}$ (about $4 \mathrm{v}$ is used, to allow for forward drop in the diodes), is nonconducting because it is held in a reverse bias condition by the action of the large negative voltage $-V$. The upper diode is conducting, meanwhile, and sets the input to the wind potentiometer at $0 \mathrm{v}$. The anode of this diode is shown connected to +0.6 $\mathrm{v}$, a value adjusted in operation to compensate for the diode forward drop so that the wind-potentiometer output actually is 0 . As the $h^{*}$ voltage rises, the voltage at the arm of the altitude breakpoint potentiometer will at some time, depending on its setting, rise above $0 \mathrm{v}$. While it is between 0 and $+5 \mathrm{v}$, both diodes are nonconducting, and a voltage builds up across the wind potentiometer, reaching a nominal value of $+5 \mathrm{v}$ before being limited by conduction in the lower diode.

The output of the wind potentiometer drives one side of a sine-cosine potentiometer. An intermediate buffer amplifier is necessary to prevent electrical loading of the wind potentiometer, and a 1:1 inverter amplifier is necessary to drive the other side of the sine-cosine potentiometer. $R_{1}$ is necessary for electrical reasons to prevent excessive current being drawn from the breakpoint potentiometer at high settings, and to standardize the forward current through the diodes. Resistors $R_{2}$ are identical summing resistors feeding ordinary analog summing amplifiers, a total of 20 connected to the east-west summer, and 20 to the north-south summer. The altitude breakpoints are set to occur at 20 steps of the $h^{*}$ voltage, equally separated from 0 to $+95 \mathrm{v}$ (for an $h^{*}$ amplitude of $100 \mathrm{v}$ ). The value of the nominal $+5 \mathrm{v}$ supply is adjusted to make the individual steps add up properly. The 20 rheostats labeled $R_{3}$ are calibration adjustments to correct for component variations, thus making the full-scale contributions from all the wind potentiometers equal.

As front panel adjustments, the $R_{3}$ rheostats could be used to weight the winds so that the 20 layers are equally spaced heights, rather than irregular height intervals spaced at equal intervals of fall time (for a selected particle size). This may prove to be desirable for convenience and flexibility. If this is done, the settings must be changed when shifting to a problem with quite different initial cloud height, or choice of typical particle.

Additional sawtooth voltages are generated by the particle slowness modulators. Each modulator produces four sawteeth while the height sweep scans through each cloud layer. The amplitudes of these sweeps are scaled according to the relative time of fall. At the midpoint of the sweep, for example, the vector sum of the outputs of the $x$ and $y$ channels is half the full amount. The result is to trace a radial line, or "spoke," that is the locus of fallout of particles of various kinds up to the slowest-falling type. These spokes are nominally constant-height lines, but are curved somewhat, because there is a little variation in height, and hence in mean wind, during the scan of particle slowness. The effect is to multiply each component by the sawtooth voltage, but no conventional multipliers are used. The effect is achieved by use of voltage-controlled sweep amplitudes, of whatever polarity is dictated by the wind pattern. These modulators are actually integrating circuits, using operational amplifiers. They are reset 800 times per second by triode reset circuits that require about $20 \mu \mathrm{sec}$.

To take into account the diameter of the cloud, the spot on the cathode-ray tube must be effectively enlarged to have a diameter proportional to that of the cloud, changing with the voltage $h^{*}$, as the solution of the problem progresses. To spread the spot sufficiently, it has been necessary to resort to a highfrequency spiral raster, with a circumferential frequency of about $100 \mathrm{kc}$ and a repetition rate (raster rate) that varies from 4 to $20 \mathrm{kc}$ or more. The highest frequency is associated with the smallest spiral diameter, because the spiral advances outward at a fixed rate until it reaches a specified voltage representing the cloud diameter. Thus, larger raster 
diameters will be repeated at a lesser rate, and will have more turns in the spiral. The computer unit is called the cloud raster generator. It is driven by a voltage from the cloud diameter generator, which is a conventional diode function generator approximating a function of one variable (the $h^{*}$ voltage) by means of connected line segments having adjustable slopes and breakpoints. The $x$ and $y$ voltage components of the spiral are inserted into the final $x$ and $y$ driver amplifiers as increments to the basic fallout positioning voltages.

The cloud activity generator receives the $h^{*}$ and $s$ voltages and continuously generates an output that is a function of these two variables. The output of this unit can be displayed on the main cathode-ray tube when desired, in the form of a family of curves of activity $\alpha$ versus slowness with $h^{*}$ as a parameter. Actually, 80 curves appear in the family, but the operator sets in curves only at the top of the cloud, ground level, and four intermediate points at equal intervals of $h^{*}$. Each curve is made up of five line segments. This generator must be unusually fast in operation, because $s$ sweeps through its entire range of $50 \mathrm{v}$ in $1 / 800 \mathrm{sec}$. Furthermore, it should be possible to adjust to arbitrary functional variations between curves in the family, rather than use polynomial methods. It is desirable that the operator simply adjust a row of knobs until the proper function of activity versus slowness is obtained at ground level, then independently adjust to match a specified curve at the next selected height in the cloud, and so on to the top of the cloud.

Function generators developed by the George A. Philbrick Co. were employed in the first two computers. They permit such independent arbitrary adjustment. However, the interpolation between curves for a constant value of the $s$ variable is not linear. For example, in progressing from a horizontal line to one with a V-shaped notch, the intermediate curves are truncated, with flat sections, rather than $V$-shapes of intermediate height. The device is all electronic, using a matrix of selector diodes. Better interpolation is desired, and an electronic function generator ${ }^{4}$ recently developed by L. Taback of the Bureau may find application in later models of this computer. The Taback generator uses a time-division technique for interpolation between curves in a family.

The output voltage of the activity generator is connected to the intensity grid of the final display cathode-ray tube. It thereby modulates the electron beam so as to make the brightness proportional to the activity of the particle type at the initial height under consideration at any instant. The intensity level of the cathode-ray tube does not remain sufficiently constant (at a specified grid test voltage) for a permanent type of calibration. Furthermore, the luminance versus grid voltage curve departs considerably from linearity in some regions of operation. Hence, slope and brightness-level controls

${ }^{4} \mathrm{~L}$. Taback, A function generator for two independent variables, Control Eng. 3, 129 (1956).

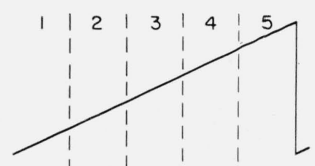

$h^{*}$, SAWTOOTH VOLTAGE, PERIOD I/IO SEC, STANDARD AMPLITUDE

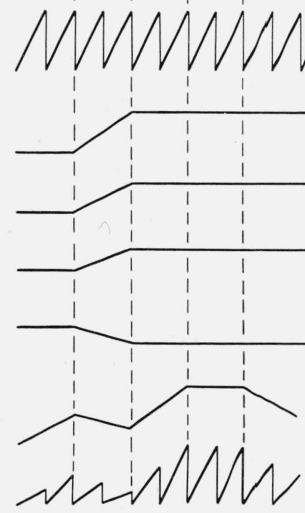

S,"SLOWNESS", ACTUAL PERIOD I/8O0 SEC, NOT AS SHOWN

INPUT TO WIND POT. NO 2

OUTPUT OF WIND POT NO.2, 2/3 MAXIMUM

NORTH OUTPUT OF SIN-COS POT. NO 2

EAST OUTPUT OF SIN-COS POT. NO.2

OUTPUT OF EAST WIND SUMMER

OUTPUT OF EAST PARTICLE SLOWNESS MODULATOR

Figure 5. Basic waveforms.

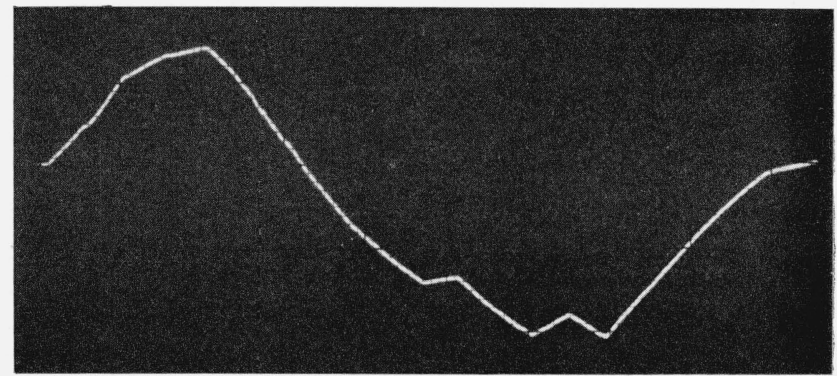

Figure 6. Output of north-south winds summer.

are provided. The slope control is an attenuator in the activity signal line. These controls must be adjusted with the entire computer in operation after insertion of a calibration problem with known winds, cloud geometry, and activity model. The procedure is to first set the $x$ and $y$ deflection factors for the cathode-ray display so as to make the display read correctly in miles for the eastward and northward limits of the pattern. For this purpose the test problem can be made very simple. Then the over-all size factor for the cloud is adjusted to make the mushroom diameter appear to be the proper number of miles on the screen. The slope and brightness-level controls for the cathode-ray tube are then adjusted to make the photometer read as correctly as possible in areas with various ranges of brightness.

Some of the basic waveforms in the computer are shown in figure 5. All are exhibited on the same time scale synchronous with the $h^{*}$ voltage, but to simplify the diagram, only 5 wind channels are shown instead of the actual 20 , and only $10 \mathrm{~s}$ sweeps instead of the actual 80 during each $h^{*}$ sweep. Four of the waveforms are associated only with wind 2 , while the two waveforms at the bottom show the contributions from channels $1,3,4$, and 5 as well.

Figures 6 to 14 were taken before the first computer was completed. They do not exactly repre- 


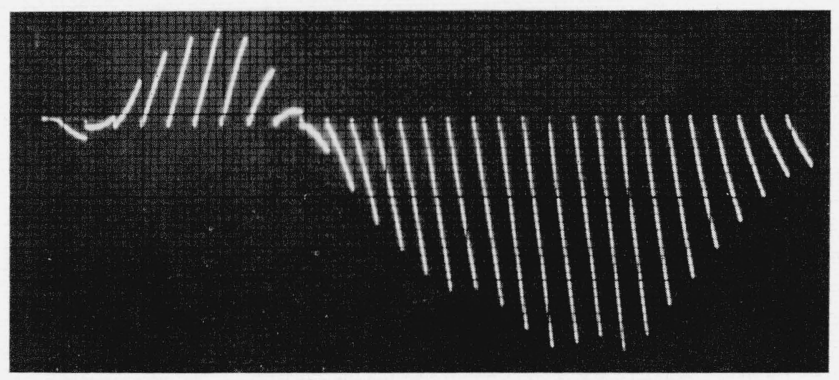

FIGURE 7. Output of north-south slowness modulator.

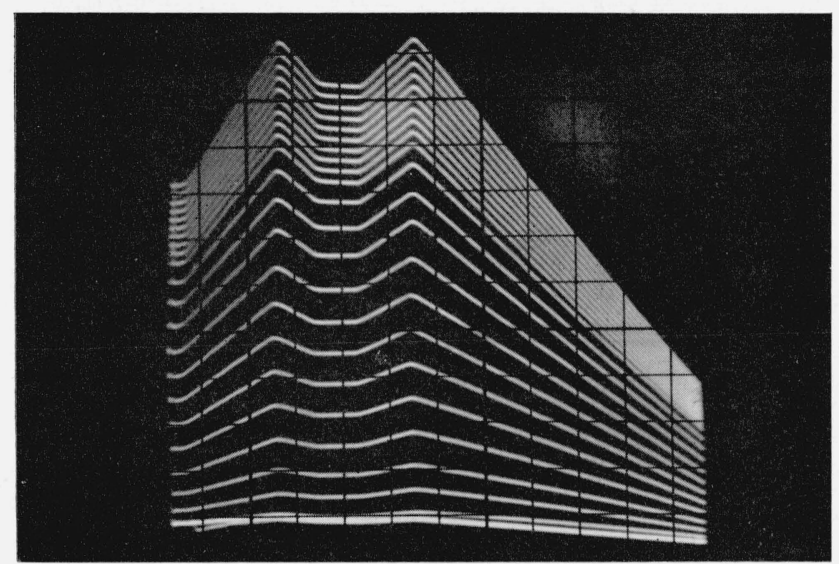

Figure 8. Output of activity generator.

sent normal operation, providing less than the normal 80 "spokes," for example. Because it was not possible to take pictures subsequently, these are presented to aid the discussion.

Figure 6 is an oscillograph trace of the output of the north-south summer versus $h^{*}$ voltage. Figure 7 then shows the result after the waveform shown in figure 6 is used to modulate the particle-slowness voltage. The triangular waveforms actually have an envelope that follows the waveform of figure 6 .

Figure 8 is an oscillograph picture of the output of the activity generator, set for convenience of display, and not to represent any actual explosion cloud. The abscissas represent particle slowness, and the ordinates represent the output activity, $\alpha$. Each of the individual curves corresponds to a different value of $h^{*}$.

Figure 9 shows the display of fallout on the cathode-ray-tube screen in the absence of any intensity modulation by the activity generator, and without the spreading of the beam by the cloud raster generator. Each spoke is the result of one slowness sweep. Fallout from successively higher initial layers in the cloud corresponds to progression of the spokes in a clockwise sense for this case. The number of spokes is determined by the ratio of the $s$ sweep frequency to the $h^{*}$ sweep frequency.

Figure 10 was photographed under the same computer settings but includes the intensity modulation according to the function $\alpha$.

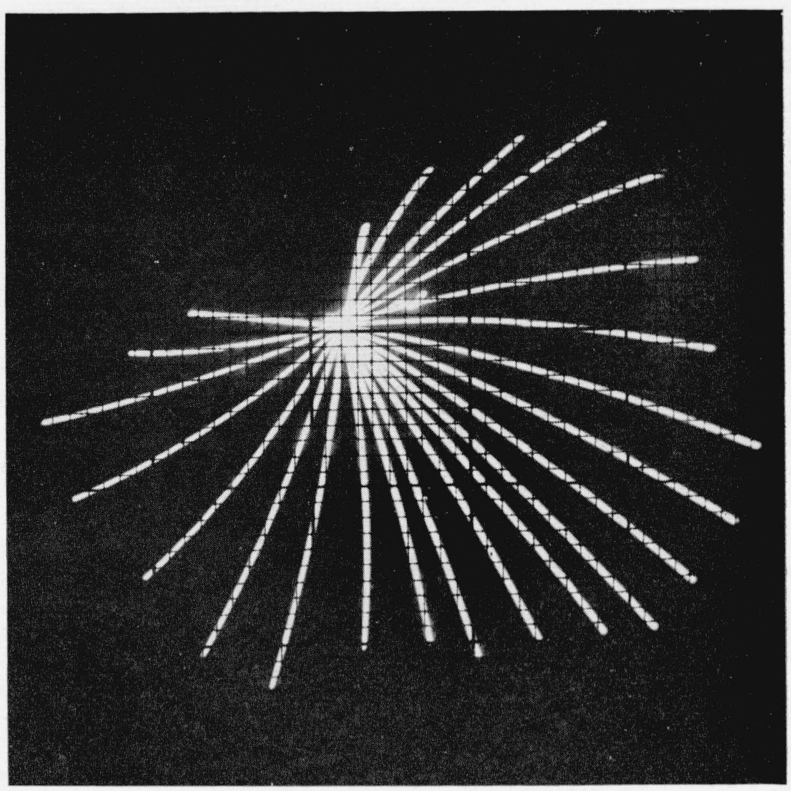

Figure 9. Simple spoke display.

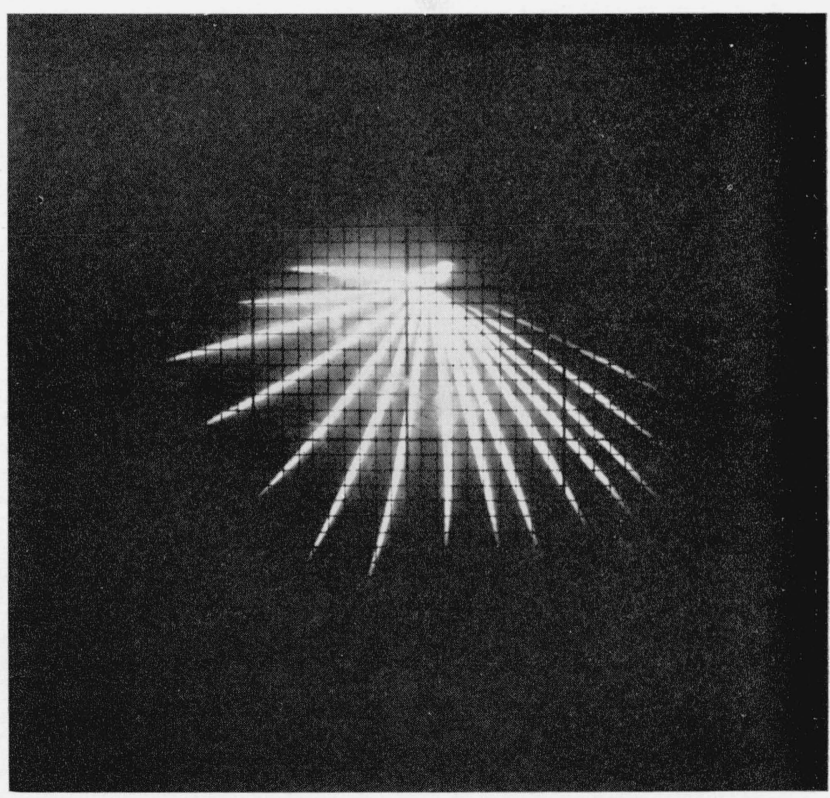

Figure 10. Intensity-modulated spokes.

Figure 11 is similar to figure 10 , except for more drastic modulation of the intensity. Here only the tips of the spokes show, as would be the case if only the slowest particles were radioactive and numerous enough to be of importance.

Figure 12 shows the result of superposing the cloud raster on the display of figure 11. In actual use, the oscilloscope spot is defocused so that the spiral pattern does not show clearly. (The appearance of spirals of widely different diameters on the same centers is not proper, and is the result of faulty synchronization existing at the time of this early picture.) 


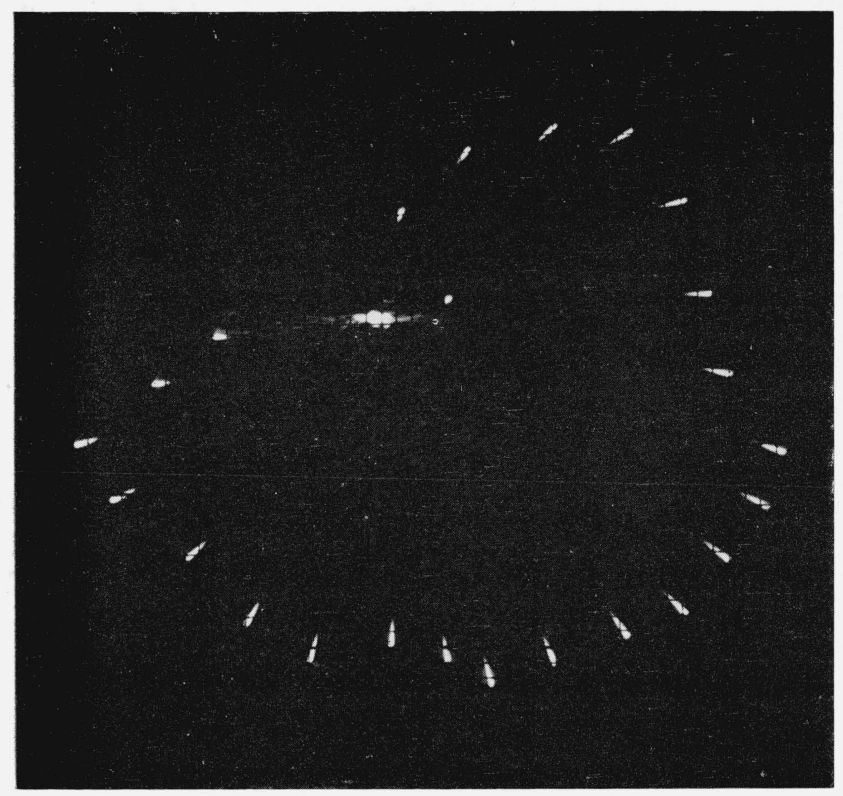

Figure 11. Spoke tips

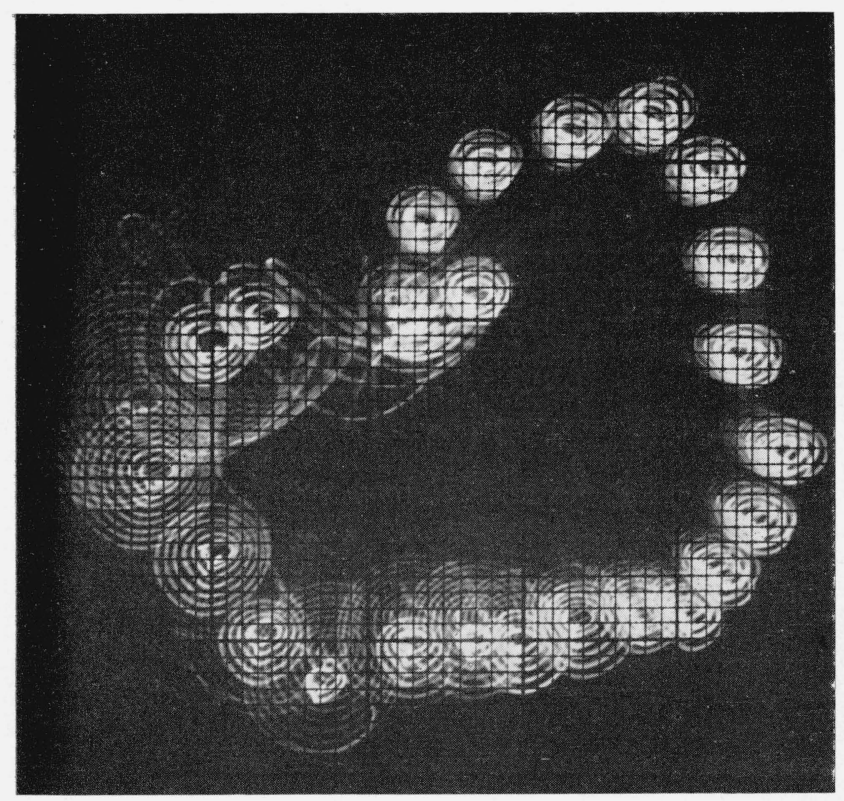

FiguRE 12. Spiral rasters.

Figure 13 shows how the final display appears with all units of the computer in action. The computer was adjusted to produce this apparent fallout without knowledge of the characteristics of any actual bomb, hence the pattern is not representative.

Figure 14 shows one of the computers. The cabinet on the right contains the computer proper, together with an auxiliary 5 -in. oscilloscope. The 4 chassis below the 5 -in. scope form the winds unit, each containing 5 wind channels. Below these, moving downward, are the cloud activity unit, central control unit, cloud diameter unit, and cloud raster

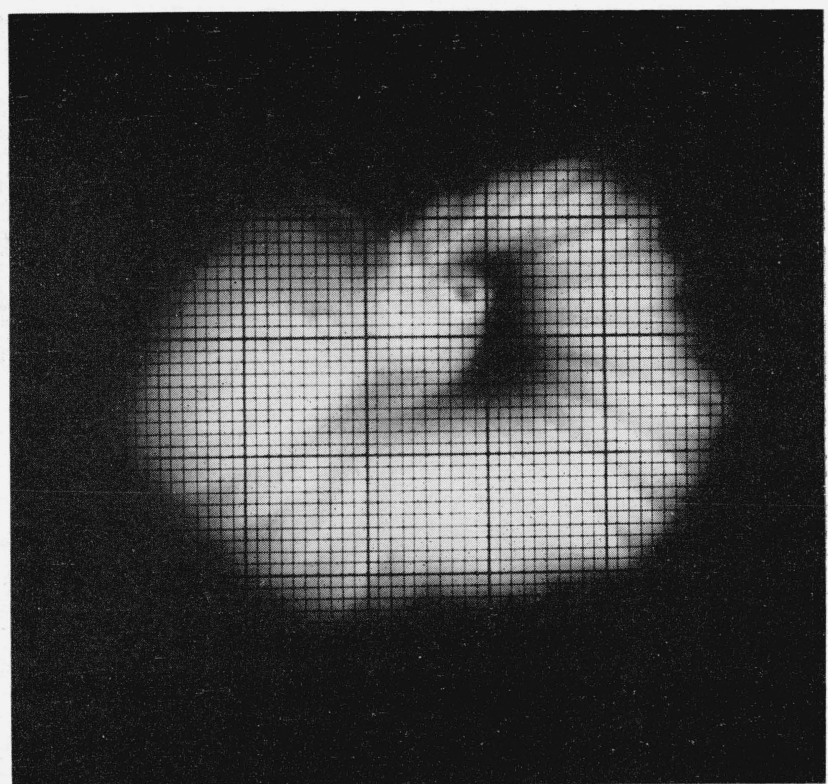

Figure 13. Complete fallout display.

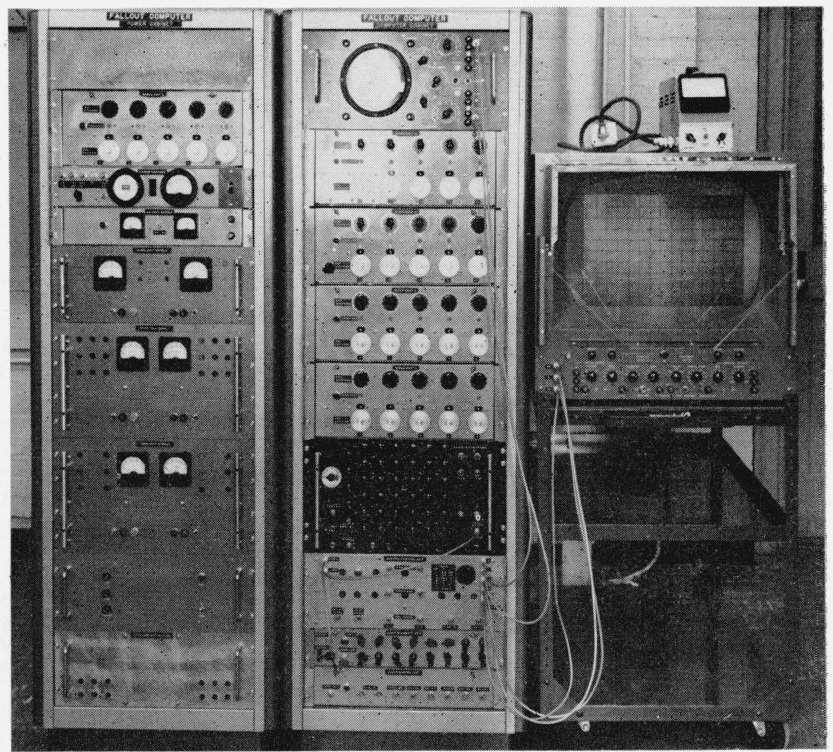

Figure 14. Computer.

generator. The 21-in. cathode-ray display unit is mounted on a table at the right. On top of it is the meter of the photometer, a modified commercial unit with a probe area $1 \frac{1}{4} \mathrm{in}$. in diameter. The probe is at the end of a cable. The cabinet at the left contains power supplies, test equipment, and a spare chassis. The second large cabinet is unnecessary, and the essential power supplies can be mounted in a small unit forming a pedestal for the display unit. Forcedair cooling is employed, with lateral airflow from front to rear over the chassis. The total a-c power requirement is about $1,500 \mathrm{w}$. 


\section{Comments on Operation}

Because the machine is used intermittently, and accuracy requirements are relatively low, chopper stabilization was not employed in the operational amplifiers. Thus, balance adjustments are necessary. These are made after $1 \mathrm{hr}$. warmup time, the process taking about $5 \mathrm{~min}$. Following this, some readjustment is necessary at intervals of 2 to $6 \mathrm{hr}$. Checks on the brightness level of the scope and balance of the activity generator are necessary at about the same intervals. A few other basic electrical adjustments are made at intervals of a few days, including sweep amplitudes and altitude breakpoints.

In use, provided the cloud geometry and activity model had already been inserted in the machine, a completely new pattern of winds could be inserted in about 2 min. once winds were specified at the proper altitudes. Results of problem modification were immediately observable on the display, but 2 to $5 \mathrm{~min}$. were required to take a useful pattern of measurements with the photometer probe.

Modification of the activity model from a set of tabular data could be done within $5 \mathrm{~min}$. The shape of the cloud could be altered as desired, but it seldom needs serious adjustment. The over-all size of the cloud could be altered instantly by turning a single knob.

Relative position of ground zero on the screen could be adjusted as quickly as desired, but with some limitations due to shortcomings of the oscilloscope. Likewise, the picture could not be expanded greatly, because of the limitation in cathode-ray deflection speed to about $0.6 \mathrm{in} . / \mu \mathrm{sec}$. This meant that the mushroom could not appear as more than $1 \frac{3 / 4}{4}$ in. in diameter on the screen, due to the peripheral rate of 100,000 turns/sec in the spiral. An overlay map on transparent backing was used over the display screen.

\section{Cathode-Ray Tube Problems}

Because of "overlap," any elementary area on the cathode-ray screen may receive many contributions of beam current during any one computation cycle. After one "strike" by the beam, others will occur as the spiral raster moves on out along a given spoke, or along an adjacent spoke. It might seem desirable to use a phosphor screen having long persistence, so that it would integrate these contributions. However, the accumulated light-energy output versus electrical charge resulting from the various strikes is not a correct integral. The relation between beam current, exposure time, and previous history is quite complicated and unsatisfactory. Storage tubes and dark trace tubes were considered, but the practical choice was a short-persistence tube. Ideally, the persistence should be negligible. The summation is accomplished in the averaging action of the photometer deflection. Because visual display was desired, the computer repetition rate had to be fast enough to reduce the flicker in visual effect. A compromise of 10 cps was made in this computer, although $30 \mathrm{cps}$ would be necessary for really substantial reduction of flicker. Actually, the phosphor was a fairly severe compromise, being a $\mathrm{P}-4$ type. Theoretically, a considerably faster type would be desirable.

\section{Refinements}

Fairly obvious engineering refinements will reduce size and improve stability, particularly in choice of components. The number of components can be reduced, and the winds unit, in particular, could be transistorized. Zener voltage diodes can be used to simplify the winds unit and eliminate the lowvoltage precision power supply.

Greater accuracy and usefulness under some conditions could be achieved by eliminating the cathoderay tube altogether. The same basic circuits would be used, but could be run much slower, thus reducing the basic problem of achieving a sufficiently high raster rate relative to the slowness sweep. The output of the activity generator would be gated into an averaging circuit when and only when $x$ and $y$ simultaneously have values falling within a specified elementary geographical area. For any such electrically probed area, the gate would open many times during each solution time, due to the overlapping previously discussed. The output would be read directly on a meter, or could be printed out on a standard electric typewriter operating from a relatively slow analog-digital converter. The presentation could have the form of a map, each advance of the typewriter representing a standard number of miles in a routine scan of the fallout area.

The present computer design could be extended to accommodate more elaborate formulation of the problem, such as time or space variability of winds. However, it is not known whether it is entirely desirable to attempt this.

The authors are indebted to Lester Machta of the U. S. Weather Bureau, who initiated the project on behalf of the Atomic Energy Commission, and to K. M. Nagler, also of the U. S. Weather Bureau, for his assistance during the project.

Washington, September 13, 1956. 